\title{
Alimentation et facteurs de mortalité des larves de corégones (Coregonus sp.). Exemple de deux lacs de niveaux trophiques différents: les lacs de Sarnen et de Hallwil (Suisse Centrale)
}

\author{
Dominique Ponton ${ }^{1}$ et Rudolf Müller \\ Seenforschungslaboratorium der EAWAG/ETH, CH-6047 Kastanienbaum \\ Received 2 June 1988; accepted 27 September 1988
}

\begin{abstract}
Whitefish larvae (Coregonus sp.) were caught at regular intervals in 1986 and 1987 in oligotrophic Lake Sarnen, and in 1987 in eutrophic Lake Hallwil.

The food spectra of the larvae in each lake were directly related to the available prey organisms. The causes for the important decrease of the larval catches in Lake Sarnen observed in 1987 are examined. There was no significant difference in the abundance and size distribution of the principal zooplankton taxa between the 1986 and 1987 samples. The effects of a sudden cooling of the surface water layer on the survival of cold-bred whitefish laryae are discussed.
\end{abstract}

\section{Introduction}

L'intérêt porté par un nombre croissant de chercheurs pour l'étude des premiers mois de la vie des corégones en milieu naturel est dû à l'importance de la phase larvaire dans la dynamique d'une population de poissons. C'est en effet à ce stade que la mortalité est la plus élevée [29] et que l'homme intervient par des déversements parfois massifs de jeunes élevés artificiellement.

Si le nombre de travaux portant sur l'alimentation de larves de corégones dans différents milieux a augmenté ces dernières années [2, 8,9,25,27], un schéma général de l'évolution de cette alimentation n'a pu être encore dégagé malgré certaines tentatives $[12,13]$. Il semble cependant que la survie larvaire soit contrôlée en grande partie par la mortalité due à un manque d'une nourriture appropriée en quantité suffisante et réellement disponible [20].

Dans ce contexte, il paraissait intéressant de comparer les spectres alimentaires de larves ayant la même longueur, donc le même stade de développement [11], mais rencontrant des conditions biotiques et abiotiques différentes. Notre étude, destinée à compléter certaines des descriptions des spectres alimentaires des larves de corégo-

\footnotetext{
${ }^{1}$ Present address: Département de biologie, Université Laval, Québec, Qué., Canada G1K7P4.
} 
nes [27], a porté en 1987 sur le lac de Sarnen, oligotrophe, et le lac de Hallwil, eutrophe. Les conditions météorologiques particulières observées au début du mois de mai 1987, c'est-à-dire durant la période de déversement des larves dans le lac de Sarnen, nous permettent de discuter l'importance du facteur climatologie pour la survie larvaire.

\section{Matériel et méthodes}

\subsection{Les sites de l'étude}

Les deux lacs de cette étude sont situés non loin de Lucerne en Suisse Centrale (fig. 1). Ces deux lacs sont approximativement à la même altitude, le lac de Sarnen se trouvant dans une région plus montagneuse que le lac de Hallwil. Les déversements de larves vésiculées de corégones, effectués par les pêcheurs professionnels, ont lieu dès la mi-mars et jusqu'à la mi-avril pour le lac de Hallwil, à partir de début mai pour le lac de Sarnen. Dans ce dernier, les œufs sont incubés dans un système où l'eau est refroidie ce qui permet de retarder la date des déversements [10].

\subsection{Captures des larves et échantillonnage du zooplancton}

Le filet poussé ainsi que le système associé d'échantillonnage du zooplancton par pompage ont été décrits précédemment [27]. Les dimensions de l'embouchure du tuyau de pompage ont été augmentées (hauteur $20 \mathrm{~cm} \times$ largeur $2 \mathrm{~cm}$ ) et le volume pompé a été porté à $500 \mathrm{l} \cdot \mathrm{min}^{-1}$; le volume filtré restant lui de $50 \mathrm{l} \cdot \mathrm{min}^{-1}$ grâce à une dérivation. La durée de chaque trait était généralement de cinq minutes, ce qui correspond à un parcours d'une distance de 500 mètres environ. Ce temps a dû être réduit à une minute pour le lac de Hallwil lors des trois dernières campagnes du fait de la forte augmentation de la biomasse planctonique provoquant un important colmatage des filets à zooplancton aussi bien que du filet poussé. Les campagnes, d'une durée d'environ deux heures, ont toutes eu lieu en milieu de journée (midi solaire).

Les échantillons de zooplancton obtenus par pompage ont été complétés par des traits verticaux effectués entre 30 mètres de profondeur et la surface en trois points du lac de Sarnen pour chaque campagne (filet de vide de maille $160 \mu \mathrm{m}$ ).

\subsection{Paramètres climatologiques}

Lors de chaque campagne, la température de l'eau a été mesurée sur un profil vertical au point central. Pour le lac de Hallwil, ces données ont été complétées par les mesures effectuées par l'Institut fédéral pour l'aménagement, l'épuration et la protection des eaux (EAWAG).

Les données journalières concernant la température de l'air ainsi que la vitesse et la direction du vent proviennent des stations de l'Institut suisse de météorologie de Lucerne et de Buchs-Suhr près de Aarau. 


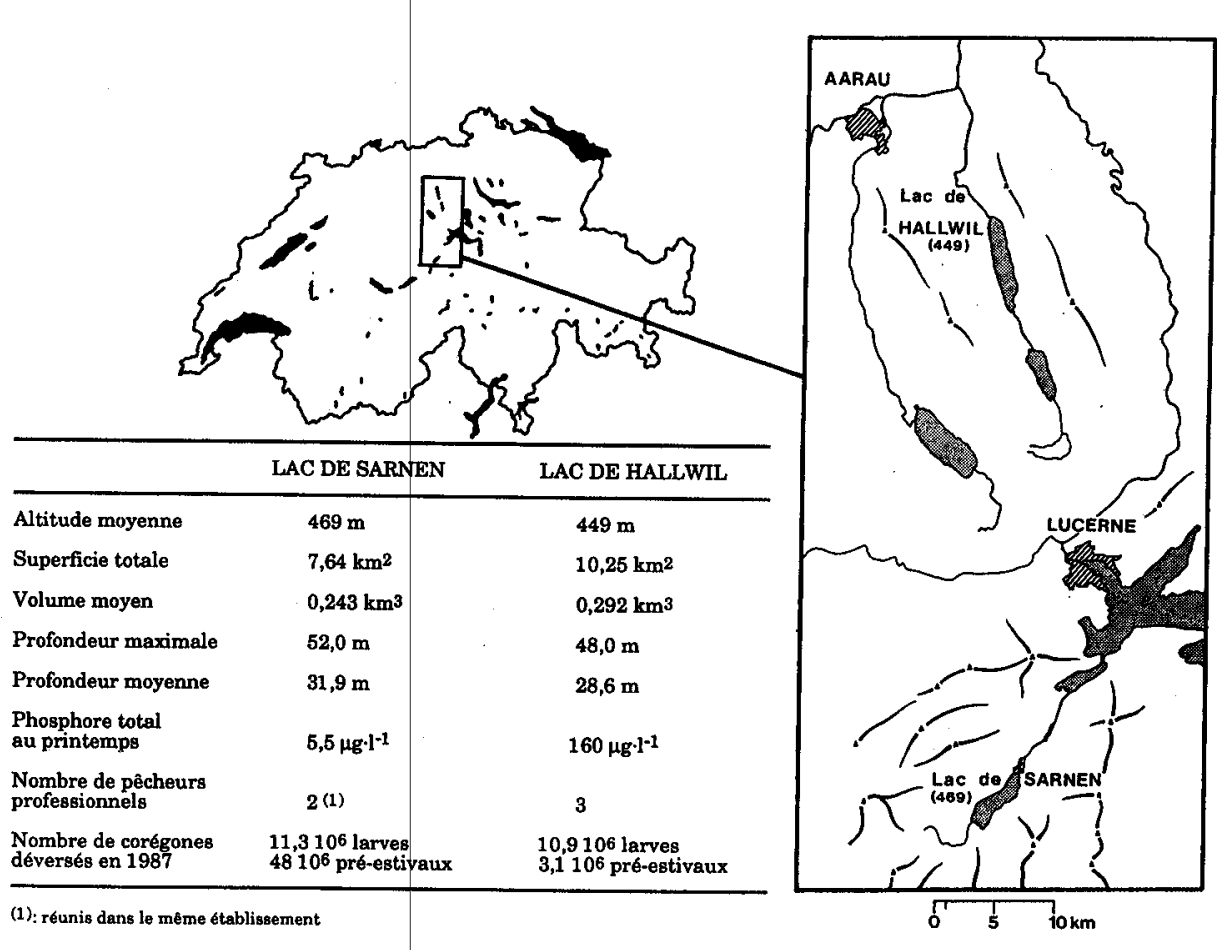

Figure 1. Situation et principales caractéristiques des lacs de Hallwil et de Sarnen. Figure 1. Location and main characteristics of Lakes Hallwil and Sarnen.

\subsection{Traitement des données}

\subsubsection{Contenus digestifs}

Les individus capturés ont été regroupés en classes de taille de $1 \mathrm{~mm}$. Seules les proies contenues dans les deux tiers antérieurs du tube digestif ont été analysées. Une conversion du pourcentage numérique de chaque taxon ingéré en pourcentage volumique a été effectuée en employant un facteur proportionnel à la taille de la proie considérée $[26,27]$. Chaque taxon proie ingéré est ainsi caractérisé par son pourcentage d'occurrence et son pourcentage volumique dans l'alimentation du groupe de prédateurs étudiés [16].

\subsubsection{Zooplancton}

Les effectifs de nauplii (de Copépodes), de Cyclopides, de Calanides, de Bosmina sp. et de Daphnia sp. ont été déterminés suivant la méthode employée précédemment [27].

Les spectres de taille du zooplancton du lac de Sarnen ont été obtenus en mesurant pour chaque échantillon 100 Cyclopides, 100 Calanides et 50 Daphnia sp. sur l'écran 


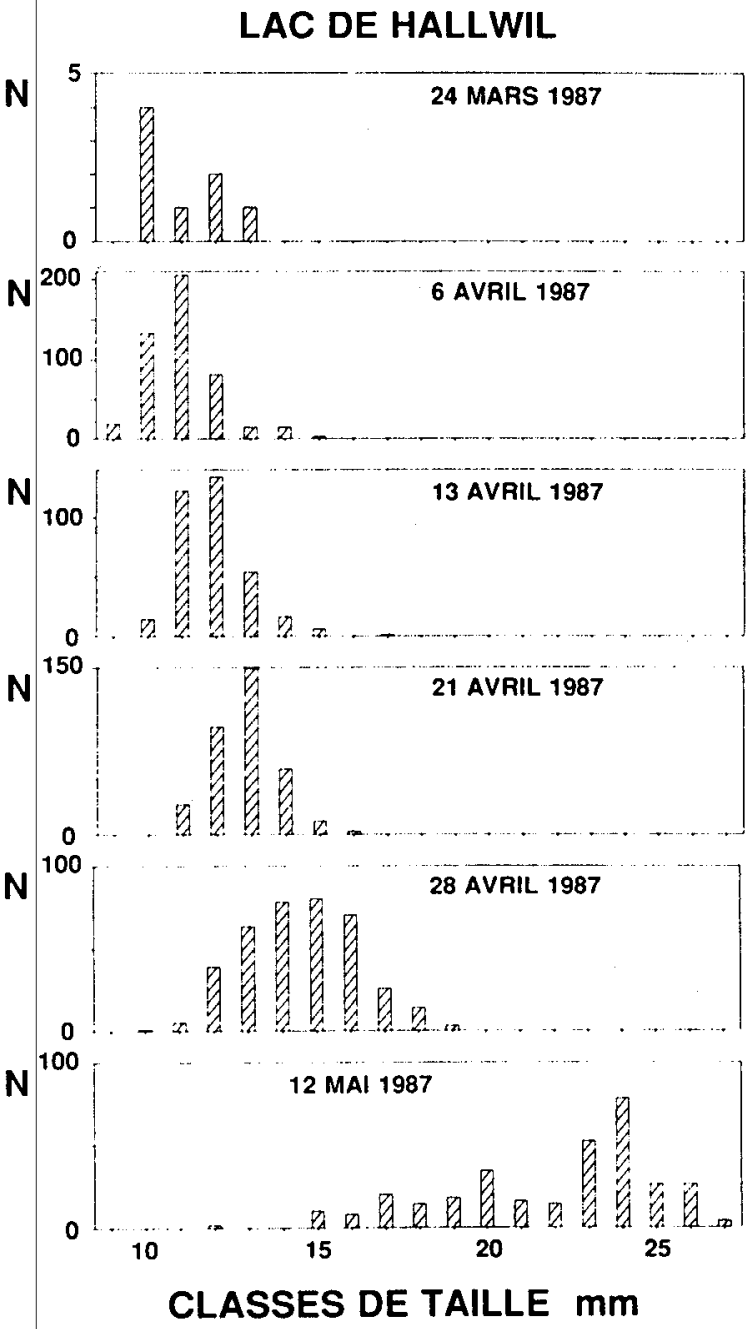

Figure 2. Répartitions en taille des individus capturés dans le lac de Hallwil en 1987. N: nombre d'individus. Figure 2. Length frequency distributions of the larvae and juveniles caught in Lake Hallwil in 1987.

d'un microscope projecteur de marque Projectina. Les individus ont été mesurés suivant les indications de McCauley [24] à l'aide d'une règle graduée. Les effectifs totaux étant connus pour chaque taxon, il est ainsi possible de déterminer les effectifs pour différentes classes de taille. 


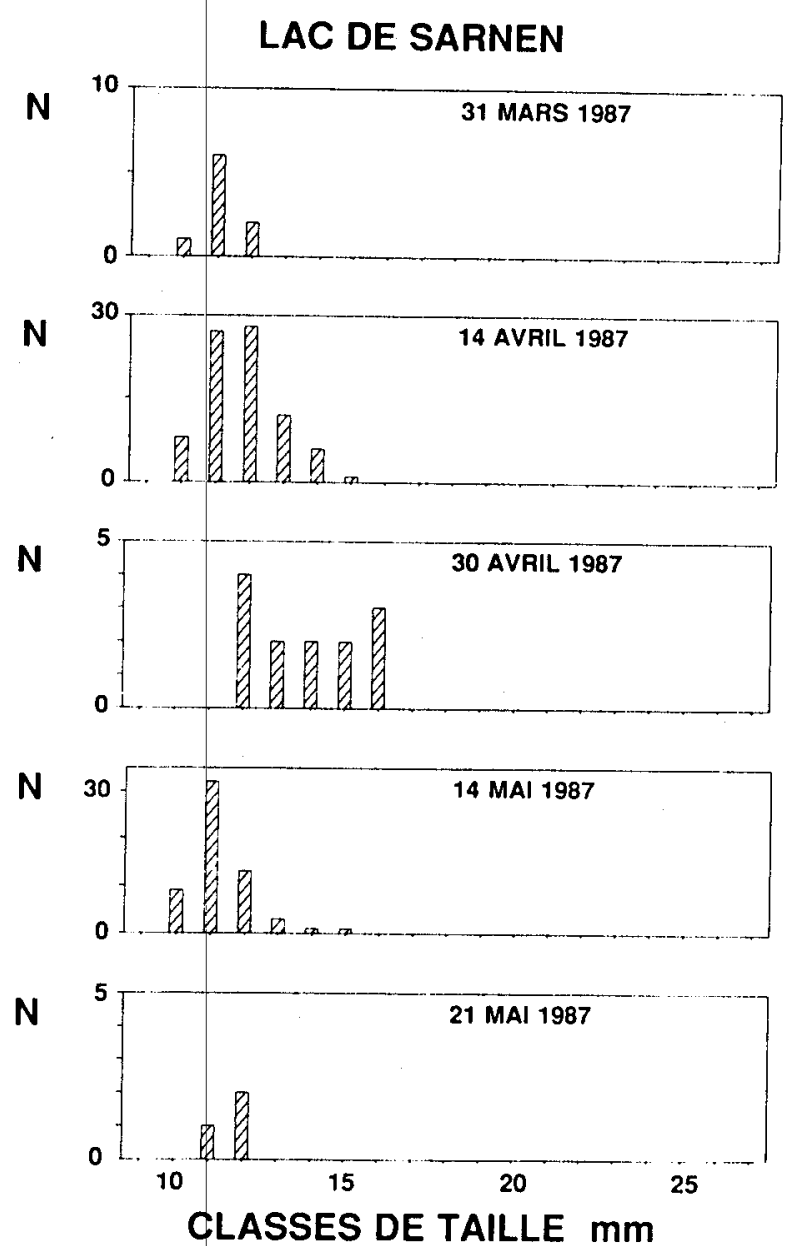

Figure 3. Répartitions en taille des individus capturés dans le lac de Sarnen en 1987. N: nombre d'individus.

Figure 3. Length frequency distributions of the larvae caught in Lake Sarnen in 1987.

\section{Résultats}

\subsection{Taille et quantité de larves capturées}

Les dispersions des distributions en taille des individus capturés au lac de Hallwil (fig. 2) sont plus élevées que celles observées dans le lac de Sarnen (fig. 3). Dans ce dernier, les déversements effectués à partir du 2 mai 1987 n'ont provoqué aucune augmentation significative du nombre de larves capturées contrairement aux déversements de mai 1986 (tabl.1). Les captures pour le lac de Hallwil atteignent par contre des valeurs très importantes (tabl. 1). 
Tableau 1: Captures de larves de corégones à l'aide du filet poussé dans le lac de Sarnen en 1986 et 1987 et dans le lac de Hallwil en 1987.

Table 1: Catches of whitefish larvae with the push-net in Lake Sarnen in 1986 and 1987, and in Lake Hallwil in 1987.

\begin{tabular}{|c|c|c|c|c|c|}
\hline $\begin{array}{c}\text { Lac } \\
\text { Année }\end{array}$ & Date & $\begin{array}{c}\text { Nombre } \\
\text { échantillons }\end{array}$ & $\begin{array}{c}\text { Nombre total } \\
\text { de larves } \\
\text { capturées }\end{array}$ & $\begin{array}{c}\text { Effort } \\
\text { total } \\
\text { en min }\end{array}$ & larves $\cdot \min ^{-1}$ \\
\hline $\begin{array}{l}\text { SARNEN } \\
1986\end{array}$ & $\begin{array}{r}17 / 4 \\
1 / 5 \\
7 / 5 \\
15 / 5 \\
22 / 5 \\
\end{array}$ & $\begin{array}{l}22 \\
24 \\
22 \\
22 \\
21 \\
\end{array}$ & $\begin{array}{r}76 \\
84 \\
639 \\
103 \\
79 \\
\end{array}$ & $\begin{array}{l}108 \\
120 \\
110 \\
110 \\
105 \\
\end{array}$ & $\begin{array}{l}0,70 \\
0,70 \\
5,81 \\
0,94 \\
0,75 \\
\end{array}$ \\
\hline $\begin{array}{c}\text { SARNEN } \\
1987\end{array}$ & $\begin{array}{r}31 / 3 \\
14 / 4 \\
30 / 4 \\
14 / 5 \\
21 / 5 \\
4 / 6\end{array}$ & $\begin{array}{l}24 \\
24 \\
22 \\
24 \\
13 \\
13 \\
\end{array}$ & $\begin{array}{r}9 \\
82 \\
13 \\
60 \\
3 \\
0 \\
\end{array}$ & $\begin{array}{r}120 \\
120 \\
110 \\
120 \\
65 \\
67 \\
\end{array}$ & $\begin{array}{c}0,12 \\
0,68 \\
0,12 \\
0,50 \\
0,05 \\
0\end{array}$ \\
\hline $\begin{array}{c}\text { HALLWIL } \\
1987\end{array}$ & $\begin{array}{r}24 / 3 \\
6 / 4 \\
13 / 4 \\
21 / 4 \\
28 / 4 \\
12 / 5\end{array}$ & $\begin{array}{l}23 \\
23 \\
21 \\
21 \\
22 \\
12\end{array}$ & $\begin{array}{r}8 \\
467 \\
347 \\
347 \\
379 \\
161\end{array}$ & $\begin{array}{r}115 \\
115 \\
75 \\
45 \\
22 \\
26\end{array}$ & $\begin{array}{r}0,07 \\
4,06 \\
4,63 \\
7,71 \\
17,23 \\
6,19\end{array}$ \\
\hline
\end{tabular}
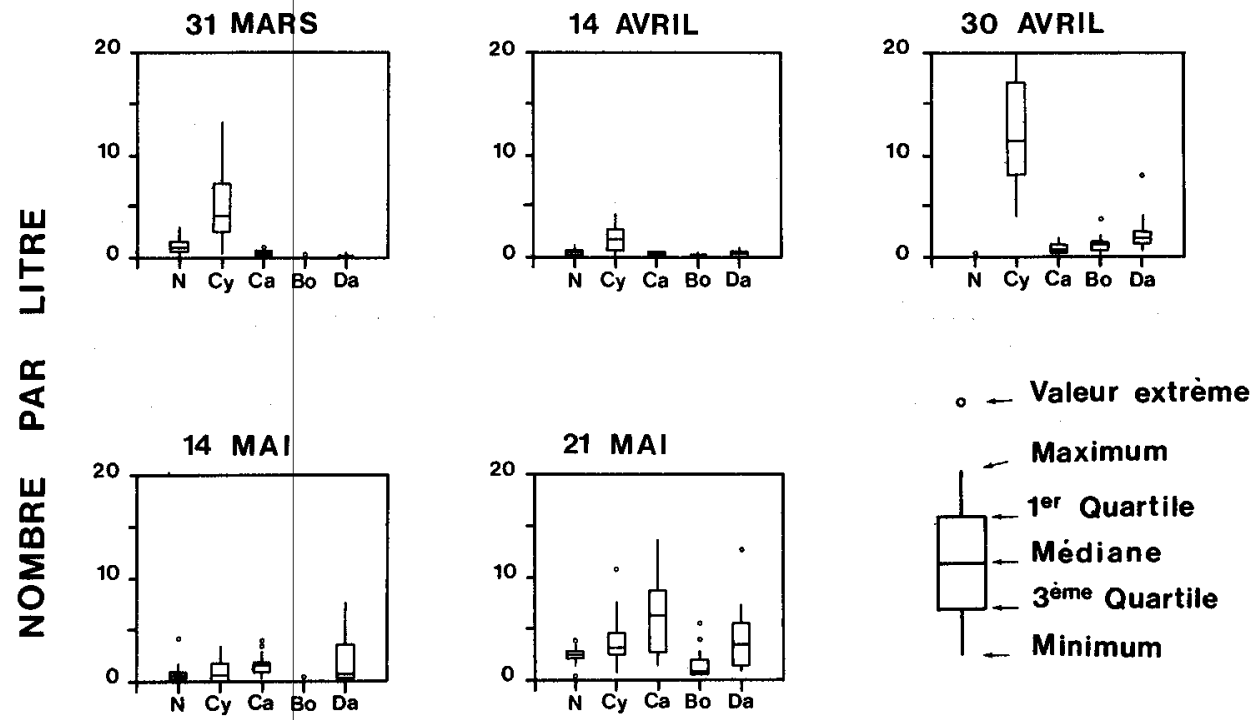

\section{TAXONS}

Figure 4. Composition du zooplancton dans la couche superficielle du lac de Sarnen en 1987. N: nauplii de Copépodes, Cy: Cyclopides; Ca: Calanides; Bo: Bosmina sp. et Da: Daphnia sp.

Figure 4. Zooplankton abundance in the near-surface layer of Lake Sarnen in 1987. N: Copepods Nauplii; Cy: Cyclopidae; Ca: Calanidae; Bo: Bosmina sp. and Da: Daphnia sp. 


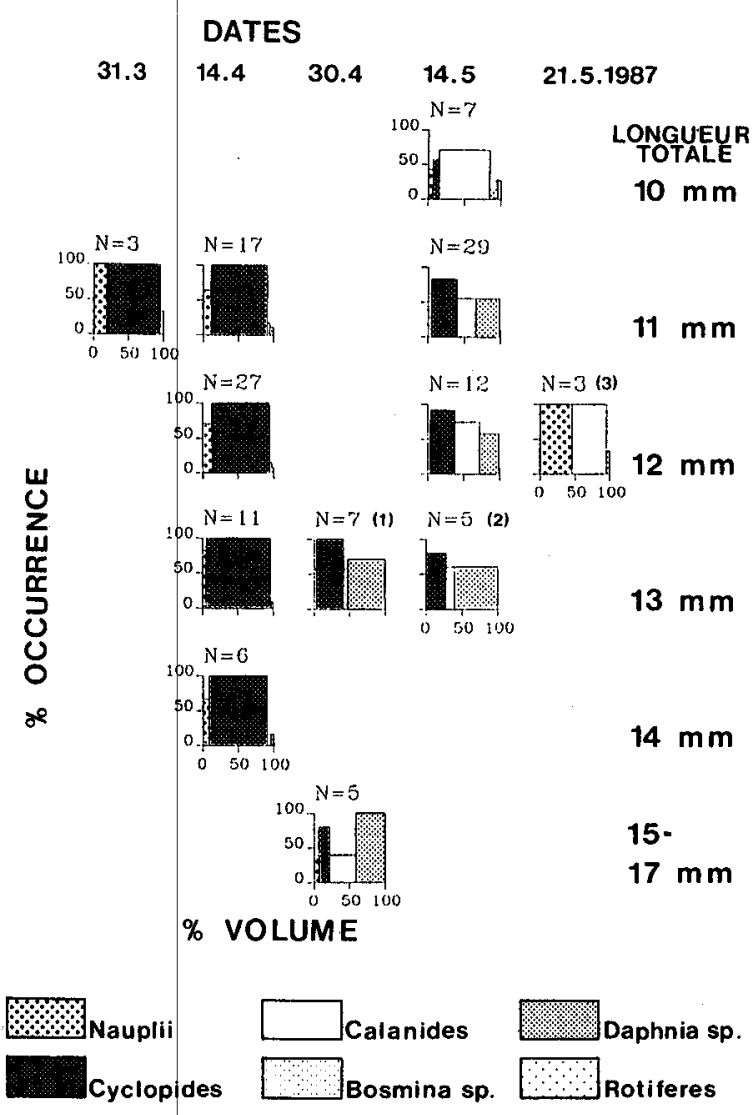

Figure 5. Spectres alimentaires des larves pour le lac de Sarnen. N: nombre d'échantillons.

${ }^{(1)}$ regroupe des individus 12,13 et $14 \mathrm{~mm} \mathrm{LT}$; ${ }^{(2)}$ regroupe des individus de 13,14 et $15 \mathrm{~mm} \mathrm{LT}$;

${ }^{(3)}$ regroupe des individus de 11 et $12 \mathrm{~mm} \mathrm{LT}$.

Figure 5. Composition of food in the gut of larvae caught in Lake Sarnen. N: number of samples.

(1) larvae of 12,13 and $14 \mathrm{~mm}$ TL together; ${ }^{(2)}$ larvae of 13,14 and $15 \mathrm{~mm}$ TL together; ${ }^{(3)}$ larvae of 11 and $12 \mathrm{~mm}$ TL together.

\subsection{Spectres alimentaires des larves dans chacun des lacs}

Etant donnée la faible quantité de matériel provenant du lac de Sarnen, les spectres alimentaires observés devront être pris avec précautions. Les différences observées dans l'alimentation des larves de corégone pour le lac de Sarnen et pour celui de Hallwil peuvent être mises en relation avec le zooplancton présent dans la couche superficielle de ces lacs au moment des captures des poissons. Ainsi, dans le lac de Sarnen, les Cyclopides sont les plus abondants en avril (fig. 4). Ils constituent alors la composante alimentaire principale des larves de corégone (fig. 5). En mai, les nauplii de Copépodes, les Calanides et Daphnia sp. sont les plus abondants, ils sont consommés de façon plus importante et le régime alimentaire des jeunes poissons est plus diversifié (fig. 5). 

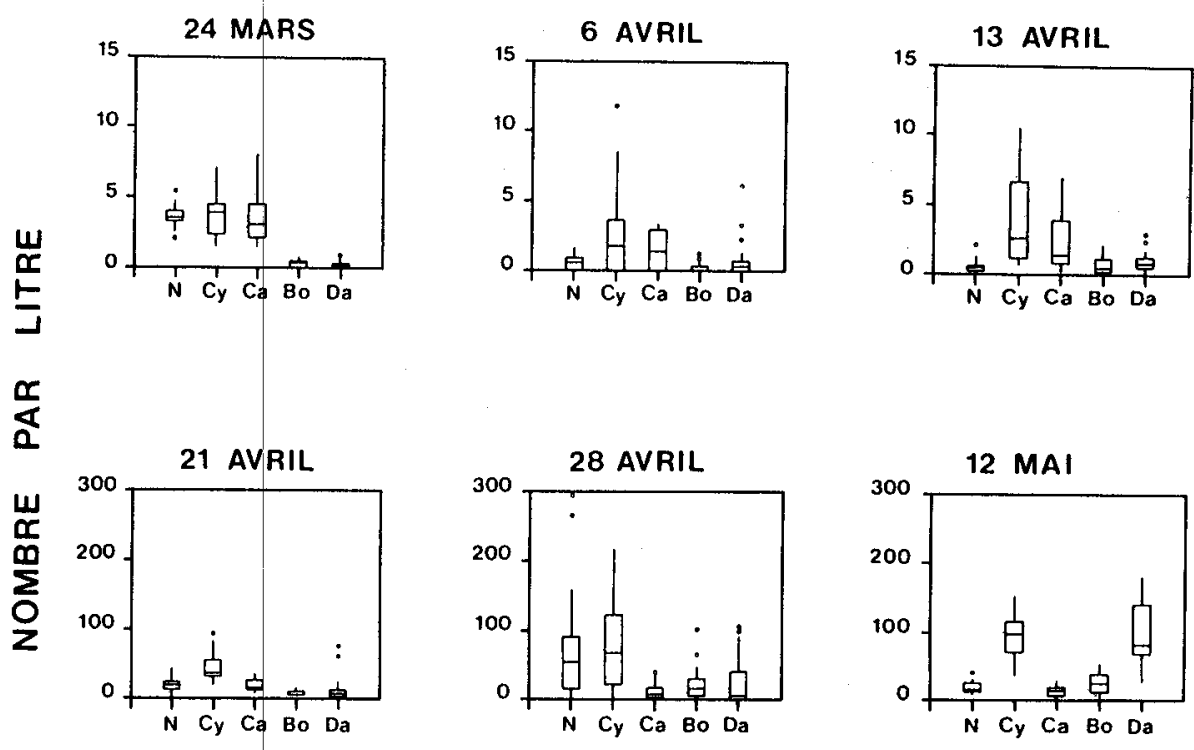

Figure 6. Composition du zooplancton dans la couche superficielle du lac de Hallwil en 1987. Légende identique à celle de la figure 4 .

Figure 6. Zooplankton abundance in the near-surface layer of Lake Hallwil in 1987.

Dans le lac de Hallwil, il n'existe pas un taxon dominant dans la couche superficielle du lac mais plusieurs associés (fig. 6): soit les nauplii de Copépodes et les Copépodes, soit les Cyclopides et les Calanides, soit encore les Cyclopides et Daphnia sp. La diversité de cette nourriture au cours du temps est bien traduite dans la diversité des spectres alimentaires observés (fig.7). On remarque pour ce lac que la forte augmentation de l'abondance du zooplancton à partir du 21 avril amène les densités des taxons à des valeurs plus de dix fois supérieures à celles rencontrées pour le lac de Sarnen.

Pour tenter d'expliquer le faible nombre de larves capturées dans le lac de Sarnen en 1987 par rapport à 1986, les abondances des trois taxons principaux consommés par les larves de corégones dans le lac de Sarnen (Cyclopides, Calanides et Daphnia sp.) ont été déterminées pour cinq classes de tailles (fig. $8 \mathrm{a}$, b et c). Quels que soient la classe de taille et le taxon considérés aucune différence d'abondance entre 1986 et 1987 n'apparaît clairement. La source de nourriture constituée par ces trois taxons principaux semble donc être la même en quantité et en qualité pour ces deux années si l'on considère le lac dans son ensemble.

Les conditions climatiques observées en 1986 et 1987 au début du mois de mai, période des déversements pour le lac de Sarnen, s'avèrent par contre très différentes. 


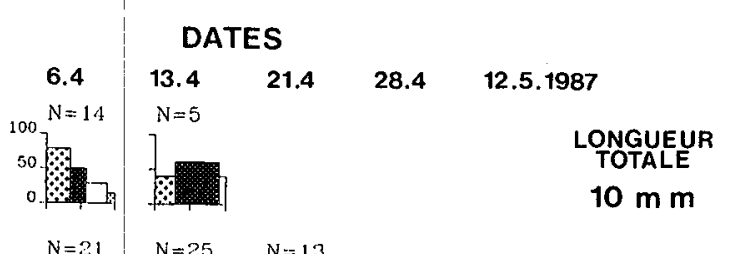

$11 \mathrm{~m} \mathrm{~m}$

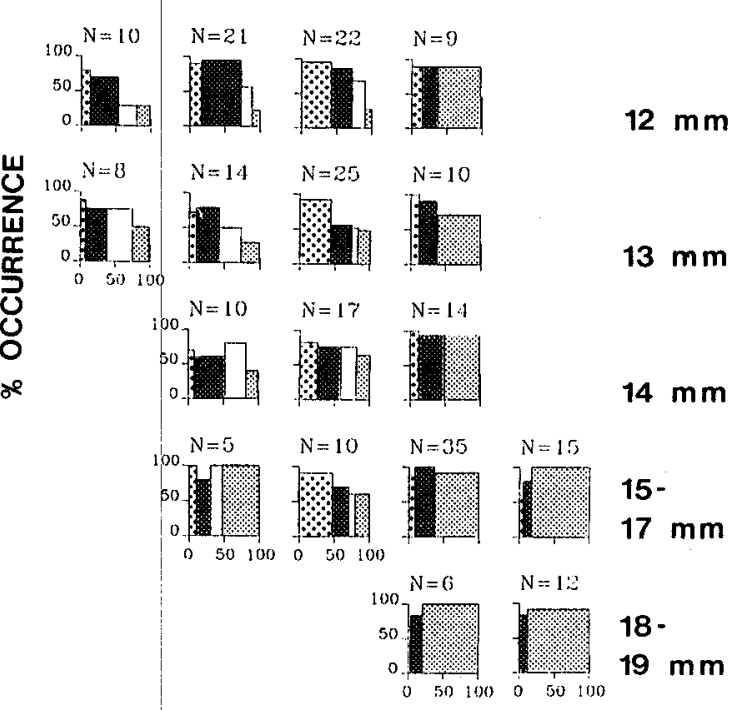

$\%$ VOLUME

Figure 7. Spectres alimentaires des larves pour le lac de Hallwil. Légende identique à celle de la figure 5 .

Figure 7. Composition of food in the gut of larvae caught in Lake Hallwil. Symbols as in figure 5.

\subsection{Comparaison des conditions climatiques observées en 1986 et 1987}

Le printemps 1987 a été caractérisé par une période de mauvais temps survenant début mai. Quelques jours de vent fort de secteur W à NNW accompagné de pluie ont provoqué une chute importante des températures moyennes journalières de l'air dans la zone d'étude par rapport à 1986 (fig. 9). Cette période de temps froid et pluvieux a provoqué une chute importante de la température de la couche d'eau superficielle des deux lacs (fig. 9). En quelques jours la température dans le premier mètre sous la surface est passée ainsi de $14-15^{\circ} \mathrm{C}$ à environ $10^{\circ} \mathrm{C}$ dans le cas du lac de Sarnen. Cette chute a été d'autant plus importante que l'augmentation de température durant le mois d'avril avait été plus rapide qu'en 1986. Une chute de la température de surface du lac de Hallwil a aussi eu lieu au début du mois de mai mais son amplitude n'a été que de $2^{\circ} \mathrm{C}$ environ. 

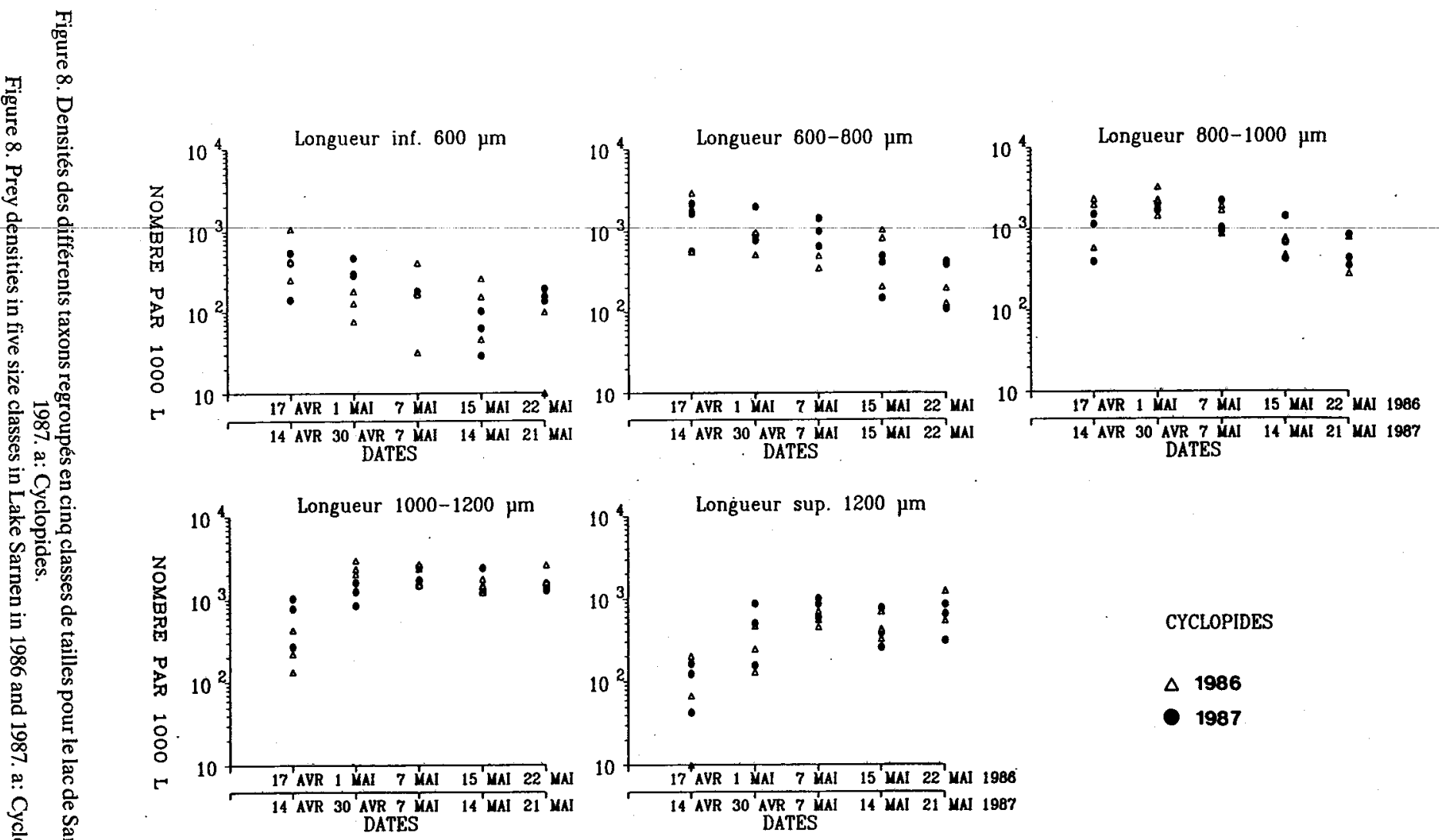

CYCLOPIDES

$\triangle 1986$

- 1987 

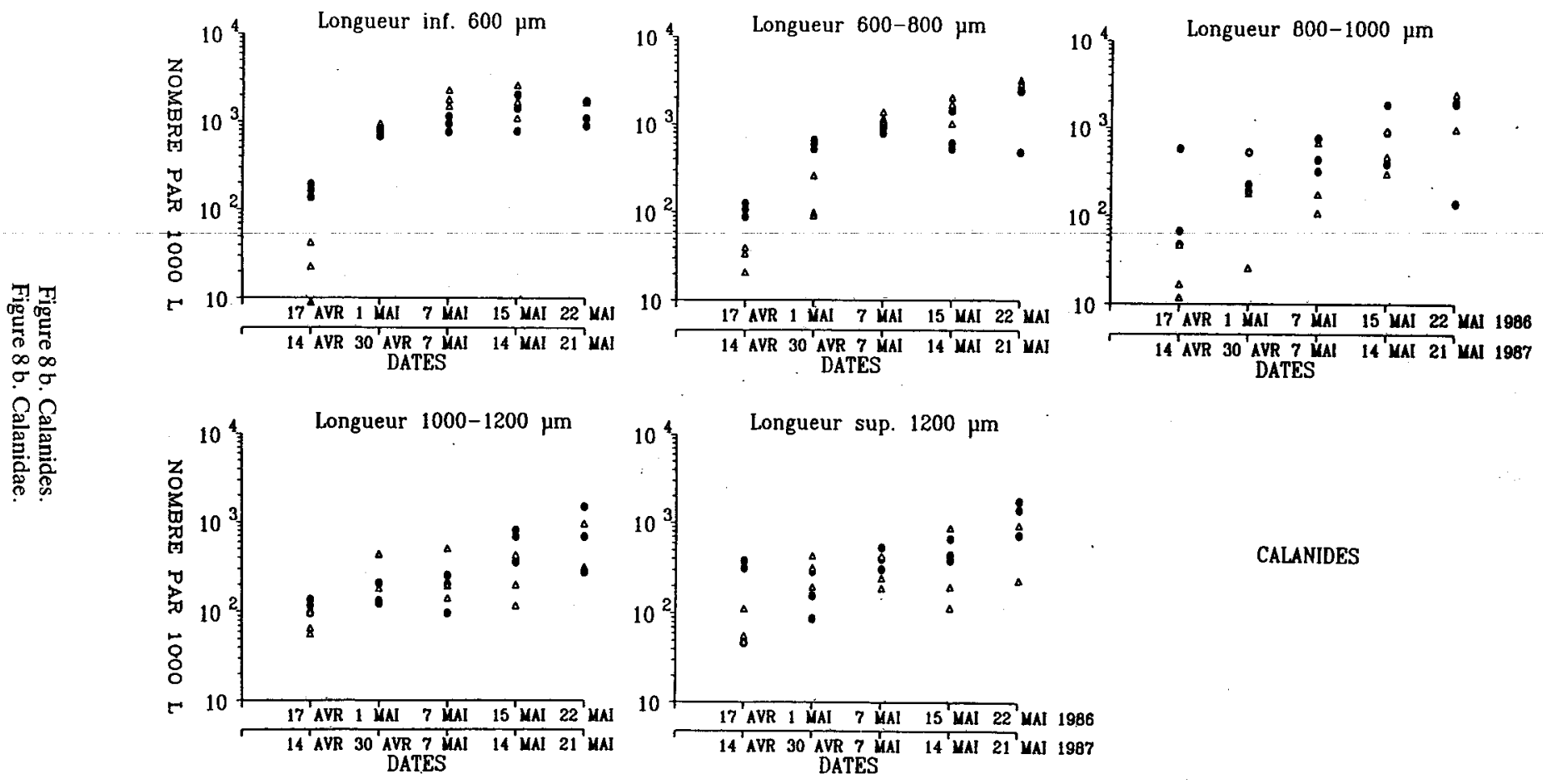

CALANIDES 

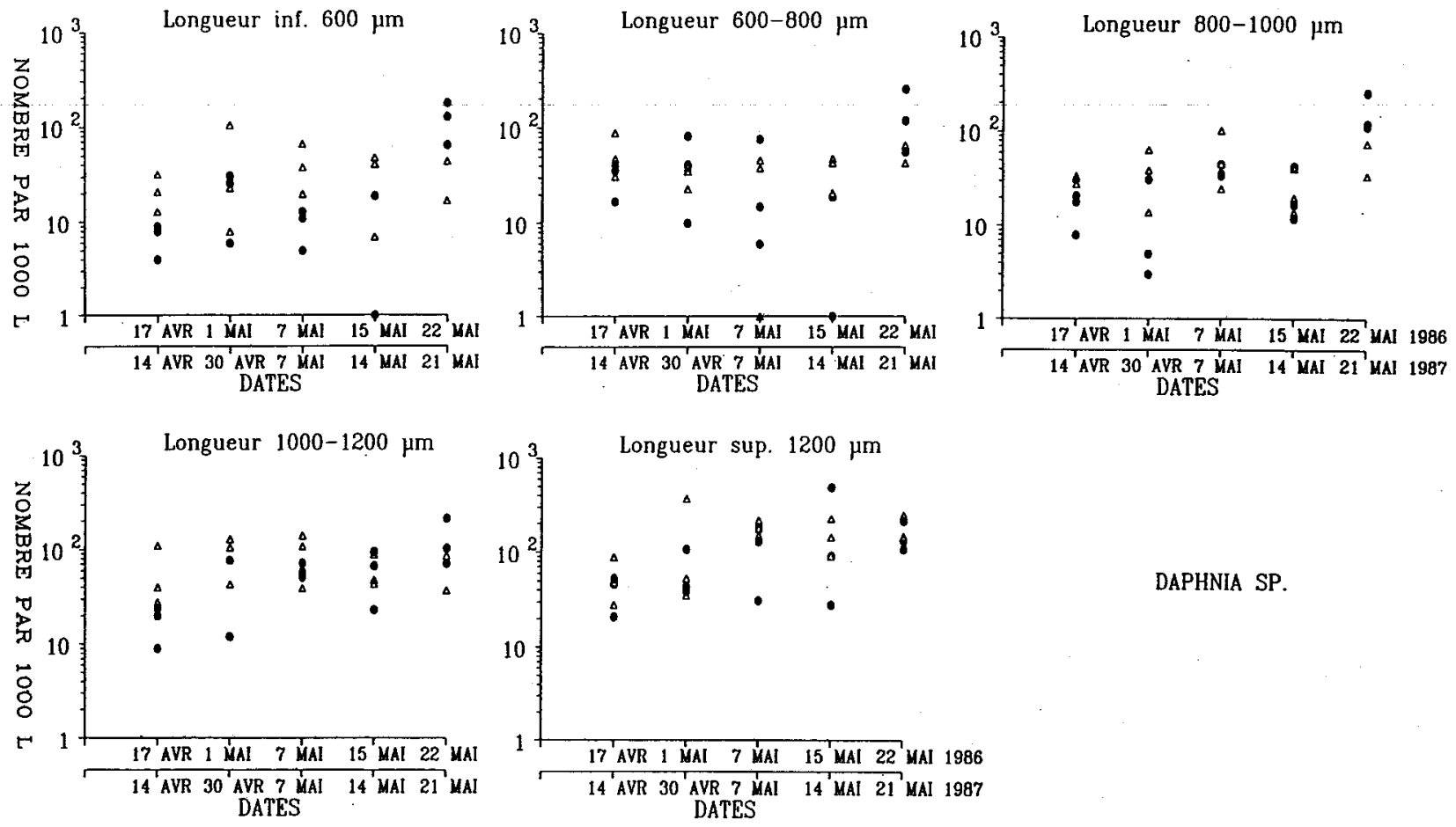

DAPHNIA SP. 
LAC DE SARNEN 1986
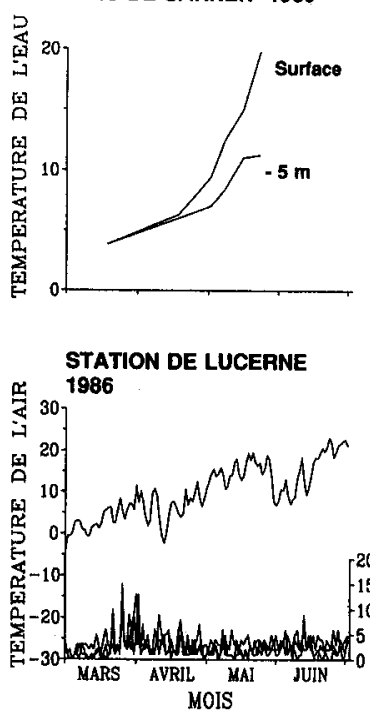

LAC DE SARNEN 1987
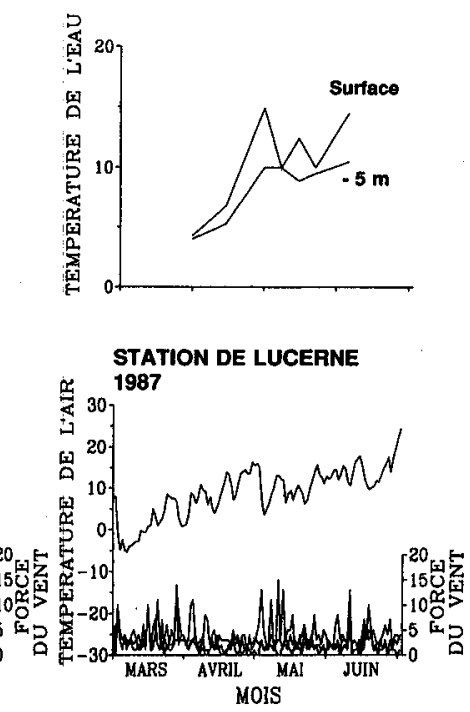

LAC DE HALLWIL 1987
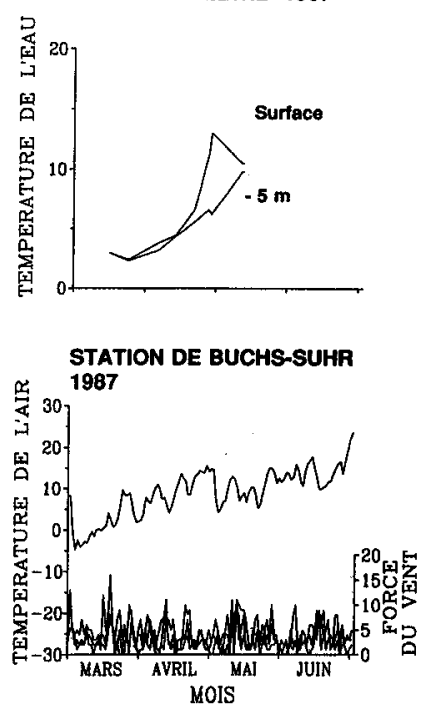

Figure 9. Températures moyennes journalières de $1^{\prime}$ 'air en ${ }^{\circ} \mathrm{C}$ et force du vent en nœuds pour les stations de Lucerne et de Buchs-Suhr (La force du vent est mesurée trois fois quotidiennement). Température de l'eau des deux lacs en surface et à 5 mètres de profondeur.

Figure 9. Daily mean air temperature in ${ }^{\circ} \mathrm{C}$, and wind speed in knots at the Lucerne and Buchs-Suhr meteorological stations (Wind speed was measured three times a day). Water temperature of the two lakes at the surface and 5 meters depth.

\section{Discussion}

Les nauplii de Copépodes et les Cyclopides constituent bien l'alimentation de base des jeunes larves de corégones. Ce fait semble être une constante pour de nombreux lacs $[8,12,13,14,21,25,27]$. La prédominance de Cyclopides dans l'alimentation est ensuite fonction de leur abondance dans le milieu. Ainsi, dans le lac de Hallwil, ce taxon est peu abondant du fait d'une circulation hivernale peu importante limitant le phénomène de levée de la diapause [5]. L'alimentation des larves s'étend donc rapidement à d'autres taxons. Daphnia sp., bien représentée dans ce lac eutrophe, constitue alors une part importante de l'alimentation des larves de corégones. On retrouve donc seulement dans ce cas la succession alimentaire du type: nauplii de Copépodes plus Cyclopides puis Cyclopides plus Daphnia sp. puis Daphnia sp. seule lorsque la taille des poissons augmente [12].

En 1987, le lac de Sarnen a surtout été caracterisé par l'absence d'une augmentation du nombre des larves capturées après les déversements effectués début mai. Le système de capture employé ne nous donne qu'une image partielle de la répartition spatio-temporelle des larves de corégone. On peut donc supposer, pour expliquer cette observation, soit un artefact dû à une distribution spatiale différente des larves, soit une réelle diminution de la quantité de larves dans le milieu par rapport à l'année précédente.

La première hypothèse semble peu probable car les captures de larves de corégone sont toujours plus abondantes près de la surface [19]. Les larves se tiennent en effet 
dans cette zone lorsqu'elles se nourrissent, ce qui leur permet de voir des proies apparaissant en contraste avec la surface lumineuse [4].

La seconde possibilité, celle d'une réelle diminution du nombre de larves dans le milieu par rapport à 1986, a l'avantage de pouvoir être étayée par de nombreux arguments.

Les larves déversées à partir de début mai proviennent d'une incubation à température basse (jusqu'à $0,5^{\circ} \mathrm{C}$ ). La durée d'incubation étant rallongée, la taille des larves à la naissance est plus élevée et leurs réserves vitellines sont en quantité plus faible $[22,23]$. Ce type d'incubation correspond en fait à un transfert du moment d'éclosion à un stade plus avancé de l'ontogénie, l'éclosion n'étant pas considérée comme correspondant à un stade de développement particulier [1].

Les larves ont habituellement des réserves vitellines suffisantes pour supporter de courtes périodes de jeûne $[6,14,17,18]$. Mais, si le stade auquel les larves sont mises dans l'environnement est plus avancé, la nécessité d'une alimentation exogène se fait sentir plus rapidement. En d'autres termes, le point de non retour en cas de jeûne est atteint plus tôt [8]. Malgré leur taille plus importante qui devrait améliorer leur capacités de nage $[3,15]$, la faible température de l'eau (environ $10^{\circ} \mathrm{C}$ ) provoque une diminution de leur vitesse de déplacement et de réactions vis-à-vis des proies [3, 4]. Le volume d'eau exploré par unité de temps pour la recherche de la nourriture ainsi que l'efficacité dans les captures se trouvent donc réduits. Comme la quantité de nourriture disponible dans le lac de Sarnen est en dessous des valeurs optimales [7] et que les petites proies de type nauplii de Copépodes ou Cyclopides de petite taille, faciles à capturer, sont peu abondantes, la ration alimentaire journalière diminue.

La basse température de l'eau et une ration alimentaire peu abondante entraînent une faible croissance. L'amélioration des capacités de nage donc l'efficacité dans la recherche de la nourriture s'effectue lentement. La période durant laquelle les larves sont vulnérables à la prédation et d'autres facteurs environnementaux défavorables est donc augmentée [21,28]. La climatologie particulière observée en 1987 a donc pu avoir une action sur la survie des larves déversées dans le lac de Sarnen. Cette hypothèse devra être confirmée par la détermination de l'abondance de cette cohorte de corégones dans la pêcherie. Dans l'affirmative, on remarquera que le refroidissement de l'eau observé en mai 1987, bien qu'agissant sur l'alimentation des larves, n'a pas eu d'effet sensible sur la qualité et la quantité de leur nourriture. Prendre comme seul paramètre des capacités de nutrition d'un milieu l'abondance en nourriture ne serait donc pas suffisant.

L'intérêt des déversements différés des larves de corégones réside dans le fait qu'elles trouvent ainsi normalement de meilleures conditions thermiques et nutritives [22]. Nous avions déjà vu que ce raisonnement était valable à condition que la nourriture soit réellement disponible dans la zone explorée par les larves [27]. Il apparaît maintenant que dans un lac oligotrophe entouré de montagnes, donc plus froid, où la quantité de nourriture reste faible durant la période des déversements, un risque existe de placer les jeunes poissons dans des conditions défavorables du fait de la fragilité de la stratification thermique facilement détruite par une courte période de mauvais temps. 


\section{Résumé}

L'alimentation des larves de corégones (Coregonus sp.) a été étudiée en 1987 dans deux lacs pré-alpins de niveaux trophiques différents: le lac de Sarnen, oligotrophe, et le lac de Hallwil, eutrophe. D'importants déversements de larves vésiculées ont lieu chaque année dès le mois de mars pour le lac de Hallwil et à partir de début mai pour le lac de Sarnen où les œufs sont incubés à basse température.

Les larves ont été capturées régulièrement entre mars et juin à l'aide d'un filet poussé, le zooplancton était échantillonné simultanément et de façon continue à la profondeur de capture des jeunes poissons.

Le large spectre alimentaire des larves du lac de Hallwil et l'alimentation principalement dirigée vers les Cyclopides de celles du lac de Sarnen, peuvent être mis directement en relation avec la nourriture disponible dans leur environnement. Les causes de la diminution drastique du nombre de larves capturées en 1987 dans le lac de Sarnen par rapport à 1986 sont examinées. L'absence de différence, tant sur le plan qualitatif (spectres de tailles des principaux taxons) que quantitatif du zooplancton présent lors de ces deux années, laisse supposer une action importante des facteurs climatiques. Les conséquences sur la survie des larves de corégones d'un refroidissement brutal de la couche superficielle du lac à la suite d'une période de temps froid et venté sont discutées.

\section{SUMMARY}

The food of Coregonus sp. larvae was studied in 1987 in two pre-alpine lakes of different trophic state, oligotrophic Lake Sarnen, and eutrophic Lake Hallwil (Fig. 1).

Abundant sac fry plantings begin each year in March in Lake Hallwil and in early May in Lake Sarnen where the eggs are incubated at low temperature. Whitefish larvae were caught with a push-net near the surface at regular intervals form March to June. The zooplankton was sampled simultaneously and continuously at the depth of larvae catches.

The wide food spectra of the larvae in Lake Hallwil (Fig.7) and the importance of the Cyclopidae in the food of the larvae in Lake Sarnen (Fig. 5) are directly related to the available prey organisms in the surface water layer of each lake (Fig. 4 and 6, respectively).

The causes for the dramatic decrease of larval catches in Lake Sarnen in 1987 by comparison with 1986 (Table 1) are examined. As there is no significant difference in the abundance and size distribution of the principal zooplankton taxa between the 1986 and 1987 samples (Fig. 8), an important role of climatic factors (Fig. 9) is to be supposed. The effects of a sudden cooling of the surface water layer of an oligotrophic lake on the survival of cold-bred whitefish larvae are discussed.

\section{REMERCIEMENTS}

Nous remercions N. Angeli de l'Institut de Limnologie de Thonon-les-bains (France) pour nous avoir prêté le système de filtration du zooplancton, ainsi que C. Grieder, K. Hauser et M. M. Bia pour leur aide sur le terrain.

\section{REFERENCES}

1 Balon, E. K.: Reflections on some decisive events in the early life of fishes. Trans. Am. Fish. Soc., 113: 178-185 (1984). 
2 Bogdanova, Ye. N., and Bogdanov, V.D.: Larval feeding of broad whitefish and tugun under natural conditions. Hydrobiol. J. 20, 102-105 (1984).

3 Braum, E.: The survival of fish larvae with reference to their feeding behaviour and the food supply. In: S. Gerking (Ed.), The biological basis of freshwater fish production, Blackwell Scientific Publ., Oxford, pp. 113-131 (1967).

4 Braum, E.: Ecological aspects of the survival of fish eggs, embryos and larvae. In: Gerking, S. (Ed.), Ecology of fresh water fish production, Blackwell Scientific Publ., Oxford, pp. 102-131 (1978).

5 Bürgi, H. R., Weber, P., and Bachmann, H.: Seasonal variations in the trophic structure of phyto- and zooplankton communities in lakes in different trophic states. Schweiz. Z. Hydrol. 47: 197-224 (1985).

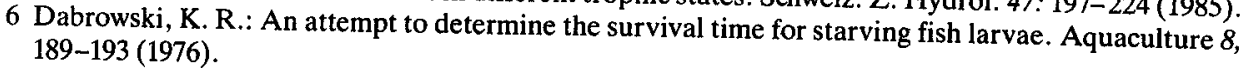

7 Dabrowski, K. R.: How to calculate the optimal density of food for fish larvae. Environm. Biol. Fish. 1, 87-89 (1976).

8 Dabrowski, K. R.: The spawning and early life history of the pollan Coregonus pollan (Thompson) in Lough Neagh, Northern Ireland. Int. Revue ges. Hydrobiol. 66, 299-326 (1981).

9 Dabrowski, K. R., Murawska, E., Terlecki J. and Wielgosz, S.: Studies on the feeding of Coregonus pollan (Thompson) alevins and fry in Lough Neagh. Int. Revue. ges. Hydrobiol. 69, 529-541 (1984).

10 Geiger, W., Meng, H.J. et Müller, R.: Méthodes de recherches en biologie de pêche dans les lacs. Nouv. EAWAG 19, 1-8(1985).

11 Gunkel, G.: Körperzusammensetzung, Wachstum und Nahrungsnutzung von jungen Sandfelchen (Coregonus fera J.) bei Fütterung mit Teichplankton und im Hunger. Arch. Hydrobiol. 92, 419-456 (1981).

12 Hartmann, J.: Two feeding strategies of young fishes. Arch. Hydrobiol. 96, 496-509 (1983).

13 Hartmann, J.: Interspecific predictors of selected prey of young fishes. Arch. Hydrobiol. Beih. Ergebn. Limnol. 22, 373-386 (1986).

14 Hoagman, W. J.: The hatching, distribution, abundance, growth and food of the larval lake whitefish (Coregonus clupeaformis Mitchill) of the Central Green Bay, Lake Michigan. Rep. Inst. Freshwat. Res. Drottningholm 53, 1-20 (1973).

15 Hoagman, W. J.: Vital activity parameters as related to the early life history of larval and post-larval lake whitefish (Coregonus clupeaformis). In: J. H. S. Blaxter (ed.), The early life history of fish, Springer-Verlag, Berlin, pp. 547-558 (1974).

16 Jacobsen, O. J.: A review of food and feeding habits in coregonid fishes. Pol. Arch. Hydrobiol. 29, 179-200 (1982).

17 Jezierska, B., Korwin-Kossakowski, M., and Jowko, G.: Influence of starvation on whitefish (Coregonus lavaretus L.) larvae. Verh. Internat. Verein. Limnol. 20, 2134-2136 (1978).

18 Jezierska, B., Korwin-Kossakowski, M., and Jowko, G.: The effect of starvation and temperature conditions of vendace (Coregonus albula L.) larvae. Pol. Arch. Hydrobiol. 26, 387-395 (1979).

19 Klein, M.: Die Beurteilung der Effektivität von Brutanstalten durch den Fang von Renkenlarven im Starnberger See. Fischer und Teichwirt 8 (1983)

20 Laurence, G. C.: Modelling: an esoteric or potential utilitarian approach to understanding larval fish dynamics? Rapp. P.-v. Réun. Cons. int. Explor. Mer. 178, 3-6 (1981).

21 Lindström, T.: Life history of whitefish young (Coregonus sp.) in two lake reservoirs. Rep. Inst. Freshwater Res. Drottningholm 44, 113-144 (1962).

22 Luczynski, M.: Improvement in the efficiency of stocking lakes with larvae of Coregonus albula L. by delaying hatching. Aquaculture 41, 99-111 (1984).

23 Luczynski, M., Dlugosz, M., Szutkiewicz, B., and Kirklewska, A.: The influence of the incubation temperature on the body length and the yolk sac volume of Coregonus albula $\mathrm{L}$. eleutheroembryos. Acta Hydrochim. Hydrobiol. 12, 615-628 (1984).

24 McCauley, E.: The estimation of the abundance and biomass of zooplankton in samples. In: A. Downing and F. H. Rigler (eds.), A manual on methods for the assessment of secondary productivity in freshwaters, IBP Handbook, vol. 17, Blackwell Scientific Publications, Oxford, pp. 228-265 (1984).

25 Niesslbeck, P. Aufwuchsbedingungen für natürlich geschlüpfte und künstlich erbrütete CoregonenLarven im Starnberger See (Obb.). Diplomarbeit Univ. Kiel. In: M. von Lukowicz (Ed.), Forschungsbericht aus der Bayerischen Landesanstalt für Fischerei, Starnberg, 145 p. (1987).

26 Ponton, D. et Stroffek, S.: Régime alimentaire des alevins de gardons (Rutilus rutilus (L.)) dans un 

port du lac Léman. Comparaison avec la nourriture disponible. Schweiz. Z. Hydrol, 49, 329-342
(1987).

27 Ponton, D., and Müller, R.: Distribution and food of larval and juvenile Coregonus sp. in Lake Sarnen (Switzerland). Finnish Fish. Res. 9, sous presse (1988).

28 Rice, J. A., Crowder, L. B., and Binkowski, F. P.: Evaluating potential sources of mortality for larval bloater (Coregonus hoyi): starvation and vulnerability to predation. Can. J. Fish. Aquat. Sci. 44, 467-472 (1987).

29 Salojärvi, K.: Spawning ecology, larval food supplies and causes of larval mortality in the whitefish (Coregonus lavaretus L.). Pol. Arch. Hydrobiol. 29, 159-174 (1982). 